\title{
Influência da corrente e taxa de alimentação na resistência à corrosão de revestimentos à base da liga Inconel 625 depositada por PTA-P
}

\author{
Influence of the current and feed rate on corrosion resistance of Inconel 625 \\ alloy coatings deposited by PTA-P
}

Breno Luíz Santos Soares ${ }^{1}$, Beatriz Cândida de Farias Ferreira ${ }^{1}$, Aureliano Xavier dos Santos ${ }^{1 *}$, Theophilo Moura Maciel², Renato Alexandre Costa de Santana ${ }^{3}$

\section{RESUMO}

O processo de exploração do petróleo ainda é muito prejudicado por falhas que ocorrem nas tubulações, as quais atrasam o cronograma operacional de produção, gerando altos custos de manutenção, além de riscos ao meio ambiente e à saúde. Com isso, a busca por alternativas menos onerosas para minimizar esses impactos é constante, principalmente contra falhas devido à corrosão. Uma dessas alternativas é o uso de materiais que possuem elevadas propriedades mecânicas e anticorrosivas na forma de revestimentos, podendo ser depositados por soldagem. Objetivou-se neste estudo avaliar os efeitos da corrente de soldagem e taxa de alimentação sobre a resistência à corrosão de revestimentos à base da liga Inconel 625, depositados pelo processo de plasma de arco transferido com alimentação em pó (PTA-P) em aço API 5L X70. Os parâmetros foram otimizados utilizando-se o planejamento experimental fatorial e os resultados analisados pela metodologia de superfície de resposta (MSR). Foram realizados três depósitos com sobreposição de $1 / 2$ por processo de soldagem PTA-P, seguindo de análise da diluição (D), teor de ferro (Fe) e resistência à corrosão por medidas eletroquímicas. Os revestimentos obtidos com menores valores de corrente de soldagem e taxa de alimentação apresentaram melhores propriedades anticorrosivas.

Palavras-chave: Aços ARBL, PTA-P, Corrosão.

\begin{abstract}
Oil exploration process is still greatly affected by pipeline failures, which delay the production schedule and create high maintenance costs, risks to the environment and health. Thus, the search for less costly alternatives to minimize these impacts is constant, especially against failures due to corrosion. One of these alternatives is the use of materials that have high mechanical and anticorrosive properties in the form of coatings, which could be deposited by welding. The objective of this study was to evaluate the effects of the welding current and feed rate on the corrosion resistance of coatings based on the Inconel 625 alloy, deposited by plasma-transferred arc with powder (PTA-P) process in API 5L X70 steel. The parameters were optimized using the factorial experimental design and the results analyzed by the response surface methodology (RSM). Three deposits with $1 / 2$ overlap were performed by PTA-P welding process, followed by an analysis of the dilution, iron content and corrosion resistance by electrochemical measurements. The coatings obtained with lower welding current and feed rate values showed better anticorrosion properties.
\end{abstract}

Keywords: ARBL steel, PTA-P, Corrosion.

\footnotetext{
1.Universidade Federal Rural de Pernambuco - Unidade Acadêmica do Cabo de Santo Agostinho - Cabo de Santo Agostinho (PE), Brasil 2.Universidade Federal de Campina Grande - Unidade Acadêmica de. Engenharia Mecânica - Campina Grande (PB), Brasil 3.Universidade Federal de Campina Grande, Unidade Acadêmica de Educação - Cuité (PB), Brasil *Autor correspondente: aureliano.xavier@ufrpe.br

Recebido: 15 Fev 2020 Aceito: 3 Mar 2020
} 


\section{INTRODUÇÃO}

No setor de petróleo e gás as tubulações são de suma importância no transporte e na distribuição desses insumos, contudo essas tubulações estão sujeitas a ambientes de condições extremas e podem vir a falhar. Por isso, pesquisadores e empresas estão adotando e desenvolvendo medidas corretivas para reduzir os impactos dessas falhas. A aplicação de revestimentos com alta resistência à corrosão com intuito de aumentar a vida útil de equipamentos do setor de petróleo e gás é uma das medidas que vêm sendo utilizadas'.

Entre os aços utilizados nas indústrias do petróleo e gás, os aços de alta resistência e baixa liga (ARBL) são os mais utilizados para fabricação de tubulações para transporte de petróleo. Os aços conforme a especificação API 5L X70 são, segundo o American Petroleum Institute (API), os mais usados para produção de tubulações por apresentarem propriedades como alta resistência mecânica, boa tenacidade, ductilidade e soldabilidade ${ }^{2}$. Contudo esses aços são suscetíveis ao fenômeno da corrosão. Por isso, o tipo de produto a ser transportado é um fator determinante na avaliação desse fenômeno, especialmente em juntas soldadas, em que defeitos podem surgir por causa da fadiga em pontos de alta concentração de tensão ${ }^{3}$

A corrosão é uma das principais causas de falhas em equipamentos e tubulações de plataformas de produção. O fenômeno de corrosão pode ser definido como o ataque destrutivo e não intencional de um metal, sendo esse ataque de natureza eletroquímica com início na superfície do metal ${ }^{4}$. Essas falhas prejudicam o processo de exploração do petróleo e atrasam o cronograma operacional de produção, gerando altos custos de manutenção, além de gerar riscos ao meio ambiente e à saúde. Com isso, uma das alternativas para minimizar essas falhas é a aplicação de camadas protetoras na superfície dos aços utilizados na indústria do petróleo e gás por soldagem¹.

De acordo com a literatura, vários processos podem ser empregados em soldagem de revestimento como: metal inert gas/metal active gas (MIG/MAG), arco submerso e plasma de arco transferido com alimentação em pó (PTA-P). O processo de soldagem a PTA-P vem sendo bastante utilizado para deposição de camadas resistentes ao desgaste e à corrosão em superfícies de materiais metálicos ${ }^{5}$.

Esse processo possui como princípio básico de funcionamento um arco de plasma que promove a coalescência de metais pelo aquecimento gerado a partir de um arco constrito. O processo incorpora três sistemas de gás, sendo o primeiro chamado de gás de plasma (em que normalmente se utiliza argônio), o segundo é chamado gás de proteção (em que se utiliza argônio ou misturas com hidrogênio) e o terceiro sistema de escoamento de gás é chamado de gás de transporte ou gás de arraste e é utilizado para carregar o material consumível durante a aplicação do revestimento (o gás utilizado nessa parte do sistema é o argônio ou misturas com hidrogênio).

O PTA-P apresenta como vantagens em relação aos demais processos de soldagem: baixa taxa de diluição, que é o percentual do metal de base que se dissolve junto ao metal de adição formando a composição química do metal de solda, pequena zona afetada pelo calor e alta taxa de deposição. Essas vantagens advêm essencialmente do controle dos parâmetros de soldagem que o processo possui, dos quais os principais são: vazão do gás de plasma, corrente de soldagem, velocidade de soldagem e taxa de alimentação. Os parâmetros citados possuem influência direta nas características geométricas da peça a ser soldada (largura, penetração), na diluição e no revestimento 5 . Assim, por apresentar tais vantagens, além de uma ampla variedade de seleção de materiais, isto é, aços, ligas à base de cobalto e níquel e compósitos com matriz metálica, o PTA-P é um processo para fácil obtenção de revestimentos?.

Para que os revestimentos depositados alcancem as características desejadas, um rigoroso ajuste dos parâmetros de soldagem deve ser realizado. Dentre os parâmetros de processo na soldagem por PTA podem-se destacar a corrente de soldagem e a taxa de alimentação. De uma forma geral, quanto maior a corrente maior será a penetração e a largura do cordão de solda, o que está atrelado ao aumento do aporte térmico, ou seja, à energia gerada durante o processo de soldagem. Entretanto, quanto maior a taxa de alimentação menor será a penetração e a diluição do metal de adição no metal base; nesse caso a energia gerada estará promovendo a fusão do metal de adição, podendo até surgir descontinuidades no revestimento, caso ocorra a elevação da taxa de alimentação $0^{6,8,9}$.

Em geral, esses revestimentos podem ser de aços inoxidáveis austeníticos ou ligas de níquel (Ni). Inconel é uma liga à base de níquel usada em ambientes extremos devido a sua alta resistência e estabilidade em condições de temperaturas extremas. A Inconel 625 é particularmente útil em várias aplicações na indústria aeroespacial e marítima e em turbinas a gás devido à sua alta processabilidade ${ }^{10,11}$. Também mostra excelentes propriedades anticorrosivas e é, portanto, um material promissor para a proteção de materiais mais baratos. A camada de óxido passivante que se forma na superfície da liga Inconel 625 é uma estrutura em camadas, ou seja, a camada interna é composta por um óxido de cromo-níquel e a camada externa composta por um óxido de molibdênio-cromo ${ }^{12}$.

Estudos descrevem o comportamento da resistência à corrosão de revestimentos Inconel 625 através de análises de polarização potenciodinâmica da superfície revestida ${ }^{13,14}$. A resistência a ambientes corrosivos é atribuída a certos elementos de liga que constituem a matriz da liga. Para que os revestimentos de liga de níquel tenham bom desempenho sob a corrosão, eles devem ter o menor teor de ferro $(\mathrm{Fe})$ possível, o qual está intrinsecamente ligado ao nível de diluição do substrato no revestimento, ou seja, quanto menor a diluição, menor a probabilidade de formação de microestruturas frágeis e susceptíveis à corrosão $0^{15}$.

A corrosão que ocorre nesses revestimentos pode ser analisada com o auxílio de técnicas eletroquímicas para avaliação da resistência à corrosão como espectroscopia de impedância eletroquímica (EIE) e polarização potenciodinâmica linear (PPL) ${ }^{13}$. A EIE é utilizada para estudar o comportamento eletroquímico de materiais e interfaces e possui um princípio de funcionamento baseado na perturbação do estado de um determinado sistema ${ }^{16}$. A PPL avalia a variação do potencial de equilíbrio estabelecido por um metal devido a reações 
que ocorrem em sua superfície; a diferença entre o potencial obtido após a polarização e o potencial de equilíbrio, é designada como sobretensão ${ }^{17}$.

Para alcançar boas características do revestimento depositado no PTA-P, como largura, reforço e penetração, é importante selecionar a combinação certa dos parâmetros do processo de soldagem. No entanto isso requer muitos experimentos e a otimização experimental se torna onerosa e lenta. Portanto é necessário utilizar ferramentas convenientes para a otimização do processo, como o planejamento fatorial experimental, que permite avaliar simultaneamente o efeito de um grande número de variáveis a partir de um número reduzido de ensaios experimentais quando comparados aos processos univariados ${ }^{18}$.

O objetivo deste trabalho é avaliar o efeito da corrente de soldagem e da taxa de alimentação sobre a resistência à corrosão de revestimentos à base da liga Inconel 625 depositados pelo processo de soldagem PTA-P em aços API 5L X70.

\section{METODOLOGIA}

O material utilizado neste trabalho como metal base foi o aço ARBL API 5L X70 com 19 mm de espessura na forma de chapa plana, tendo sua composição química apresentada na Tabela 1. A chapa foi cortada pelo processo "oxicorte" para obtenção dos corpos de prova.

Tabela 1: Composição química do aço API $5 \mathrm{~L}$ X70.

\begin{tabular}{c|c|c|c|c|c|c|c|c|}
$\mathbf{C}$ & $\mathbf{S i}$ & $\mathbf{M n}$ & $\mathbf{P}$ & $\mathbf{S}$ & $\mathbf{C r}$ & $\mathbf{M o}$ & $\mathbf{N i}$ \\
\hline 0,19 & 0,24 & 0,7 & 0,018 & 0,018 & 0,064 & 0,008 & 0,07 \\
\hline
\end{tabular}

A otimização dos parâmetros do processo corrente de soldagem (I) e taxa de alimentação do pó (Tx) em estudo foi estabelecida através de planejamento experimental fatorial em dois níveis com três repetições no ponto central, conforme os dados apresentados na Tabela 2.

Tabela 2: Níveis reais e codificados dos fatores em estudo corrente e taxa de alimentação do pó.

\begin{tabular}{c|c|c|c|c|c|}
\hline Parâmetros & Unidades & Notação & -1 & \multicolumn{3}{|c|}{ Níveis } & $\mathbf{0}$ \\
\hline Corrente & A & I & 170 & 200 & 230 \\
\hline Taxa de alimentação & g/min & Tx & 25 & 30 & 35 \\
\hline
\end{tabular}

Seguindo as regras do planejamento fatorial, a sequência das quatro combinações possíveis das variáveis de entrada somadas ao ponto central realizado três vezes, resulta em sete experimentos $\left(2^{2}+3\right)$ realizados em ordem aleatória $(\mathrm{OA})$. A sequência aleatória dos experimentos permite a aplicação de testes estatísticos de significância, a construção de intervalos de confiança e a determinação da significância dos efeitos. A Tabela 3 apresenta a matriz de planejamento completa do estudo.

Tabela 3: Matriz de planejamento experimental $2^{2}$ somado à tréplica do ponto central.

\begin{tabular}{|c|c|c|c|c|c|c|c|}
\hline Experimentos & 1 & 2 & 3 & 4 & 5 & 6 & 7 \\
\hline I (A) & 170 & 230 & 170 & 230 & 200 & 200 & 200 \\
\hline Tx (g/min) & 25 & 25 & 35 & 35 & 30 & 30 & 30 \\
\hline
\end{tabular}

Foi utilizada fonte de soldagem (DIGI Plus A7, IMC Soldagem) que permite operar em diversas modalidades de soldagem, além de possuir capacidade de corrente máxima de 800 A. O gás argônio puro foi utilizado durante a soldagem para os três fluxos de gases (gás de proteção, gás de plasma e gás de arraste).

A liga de níquel Inconel 625 (AWS ER NiCrMo-3) na forma de pó atomizado, apresentando o pó na faixa granulométrica entre 60 a 63 $\mu \mathrm{m}$, cuja composição química está apresentada na Tabela 4, foi utilizada como metal de adição para o processo de soldagem por PTA-P.

Tabela 4: Composição química (\% em peso) do metal de adição na forma de pó.

\begin{tabular}{c|c|c|c|c|c|c|c|c|c|c|c|c|}
\hline$\%$ & $\mathbf{C}$ & $\mathbf{M n}$ & $\mathrm{Si}$ & $\mathbf{C r}$ & $\mathbf{C o}$ & $\mathbf{M o}$ & $\mathrm{Fe}$ & $\mathbf{A l}$ & $\mathbf{T i}$ & $\mathbf{N i}$ \\
\hline Mínimo & - & - & - & 20,00 & - & 8,00 & - & - & - & 58,00 \\
\hline Máximo & 0,1 & 0,5 & 0,5 & 23,00 & 1,00 & 10,00 & 5,00 & 0,40 & 0,40 & - \\
\hline
\end{tabular}

Alguns parâmetros do processo de soldagem foram mantidos constantes para deposição das camadas de revestimentos, conforme apresentado na Tabela 5 . 
Tabela 5: Parâmetros fixos utilizados no processo.

\begin{tabular}{|c|c|}
\hline \multicolumn{2}{|c|}{ Parâmetros } \\
\hline Recuo do eletrodo & $1 \mathrm{~mm}$ \\
\hline Diâmetro do bico constritor & $3,10 \mathrm{~mm}$ \\
\hline Polaridade & Reserva (CC-) \\
\hline Vazão do gás de proteção & $10 \mathrm{~L} / \mathrm{min}$ \\
\hline Vazão do gás de plasma & $2,2 \mathrm{~L} / \mathrm{min}$ \\
\hline Vazão do gás de arraste & $3 \mathrm{~L} / \mathrm{min}$ \\
\hline Velocidade de soldagem & $8 \mathrm{~cm} / \mathrm{min}$ \\
\hline DBP & $20 \mathrm{~mm}$ \\
\hline Ângulo de ataque & $90^{\circ}$ \\
\hline
\end{tabular}

A deposição foi feita com três camadas de revestimento utilizando uma sobreposição de $1 / 2$ de um tamanho inicial (L), ou seja, a segunda camada teve início em e em seguida a terceira camada tendo início em , utilizando temperatura de interpasse de $100{ }^{\circ} \mathrm{C}$, como é ilustrado na Fig. 1.

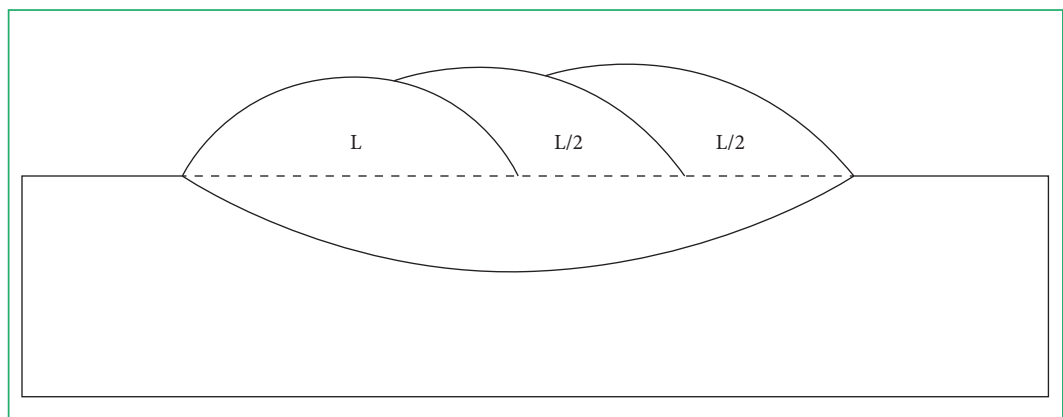

Figura 1: Esquema do sequenciamento de passes.

Uma cortadeira metalográfica foi utilizada para a confecção dos corpos de prova. Após o corte das amostras, realizou-se embutimento a frio utilizando resina poliéster. As amostras passaram por tratamento metalográfico através de lixamento. As granulometrias utilizadas foram de 100,120, 220, 320, 400, 600, 800, 1000 e 1200, com frequência de 400, 380, 360, 340 e 300 rpm, variando da menor até a maior numeração das lixas.

A análise da composição química (teor de ferro) foi feita por energia dispersiva de raios-X (EDX), utilizando equipamento Analytical QX-2000.

As amostras foram submetidas à análise de resistência à corrosão e as seguintes medidas eletroquímicas de corrosão foram obtidas: potencial de corrosão $\left(\mathrm{E}_{\text {corr }}\right)$, resistência à polarização $(\mathrm{Rp})$ e corrente de corrosão $\left(\mathrm{I}_{\text {corr }}\right)$ através da técnica de polarização potenciodinâmica linear e espectroscopia de impedância eletroquímica. O equipamento utilizado foi o potenciostato/galvanostato PG STATE302N da Autolab. O equipamento funciona com auxílio de uma placa de interface PCI instalada em um computador que possua o software NOVA 1.9 para a aquisição de dados.

A avaliação foi realizada utilizando uma célula eletroquímica no interior de uma gaiola de Faraday a temperatura ambiente. Para o meio corrosivo, foram utilizados $60 \mathrm{ml}$ de solução de $1 \mathrm{mmol} \cdot \mathrm{L}^{-1} \mathrm{NaCl}$, um eletrodo de prata/cloreto de prata (Ag/AgCl) como o eletrodo de referência, e um fio de platina em forma de espiral para aumentar a superfície de contato como contra-eletrodo. O eletrodo de trabalho foi a amostra de aço API 5L X70, revestida com a liga de níquel Inconel 625.

\section{RESULTADOS E DISCUSSÃO}

A Tabela 6 apresenta a matriz de planejamento fatorial $2^{2}$ mais três pontos centrais e os resultados experimentais das deposições por soldagem, com relação às variáveis dependentes: diluição (D\%), teor de ferro (Fe\%), $\mathrm{E}_{\text {corr }}$, $\mathrm{Rp}_{\mathrm{e}} \mathrm{I}_{\text {corr }}$, variando-se as variáveis independentes: corrente de soldagem (I) e taxa de alimentação (Tx).

De acordo com os resultados obtidos com o método experimental de planejamento fatorial, foram geradas equações (Eqs. 1-5) que representam uma linearização das superfícies de resposta, relacionando as variáveis dependentes (D, Fe, Rp, $\mathrm{E}_{\text {corr }}$ e $\left.\mathrm{I}_{\text {corr }}\right)$ em função das independentes (I e Tx). 
Tabela 6: Matriz de planejamento fatorial $2^{2}$ e resultados obtidos por PTA-P.

\begin{tabular}{|c|c|c|c|c|c|c|c|}
\hline $\begin{array}{l}\text { Parâmetros/ } \\
\text { Experimentos }\end{array}$ & $\begin{array}{c}\text { I } \\
(\mathrm{A})\end{array}$ & $\begin{array}{c}\text { Tx } \\
\text { (g/min) }\end{array}$ & $\begin{array}{c}D \\
(\%)\end{array}$ & $\begin{array}{l}\mathrm{Fe} \\
(\%)\end{array}$ & $\begin{array}{c}\mathrm{Rp} \\
(\mathrm{K} \Omega)\end{array}$ & $\begin{array}{l}E_{\text {corr }} \\
\text { (V) }\end{array}$ & $\begin{array}{l}I_{\text {corr }} \\
(A)\end{array}$ \\
\hline 1 & 170 & 25 & 4,33 & 3,64 & 486,95 & $-0,562$ & 5,70 \\
\hline 2 & 230 & 25 & 13,82 & 10,62 & 344,30 & $-0,608$ & 18,05 \\
\hline 3 & 170 & 35 & 5,59 & 4,73 & 364,91 & $-0,578$ & 7,31 \\
\hline 4 & 230 & 35 & 16,20 & 12,88 & 324,58 & $-0,641$ & 21,17 \\
\hline 5 & 200 & 30 & 10,20 & 7,97 & 346,99 & $-0,601$ & 13,32 \\
\hline 6 & 200 & 30 & 10,25 & 8,27 & 344,30 & $-0,606$ & 13,39 \\
\hline 7 & 200 & 30 & 10,17 & 6,94 & 338,99 & $-0,591$ & 13,28 \\
\hline
\end{tabular}

\section{Influência das variáveis com relação à diluição}

Com base na Fig. 2(a), verifica-se que a corrente de soldagem (I) e a taxa de alimentação (Tx) mostraram influência estatisticamente significativa, enquanto a interação entre as variáveis $I \times T x$, não apresentou a mesma influência. Os valores positivos nas colunas da corrente de soldagem indicam que o aumento da diluição é favorecido à medida que ocorre o aumento dessas variáveis. Como afirmam D'Oliveira et al..$^{19}$, o processo PTA-P produz valores de diluição de aproximadamente 5 a $10 \%$; assim, os resultados obtidos nos experimentos 1, 3, 5, 6 e 7 demonstram que, com as devidas variações nos parâmetros do processo de soldagem, os resultados obtidos para diluição apresentam adequados e podem ser utilizados para aplicação de revestimentos.

Segundo Silva e Dutra ${ }^{5}$, devido ao aporte térmico fornecido no processo de soldagem, correntes muito baixas acarretam em falta de fusão nas laterais do cordão, mas, por outro lado, correntes altas causam excesso de diluição, o que se deve ao aumento da energia de soldagem que proporciona aumento na área fundida sobre a poça de fusão.

Resultado também observado por Bharath et al. ${ }^{20}$, que utilizaram o processo de soldagem PTA-P com uma liga à base de cromo e tungstênio para fazer revestimentos em aços (X45CrSi9-3), onde foi verificado o aumento dos níveis de diluição com o aumento da corrente de soldagem. Os autores verificaram que os níveis de diluição devem ser mantidos baixos, para que um revestimento seja eficaz em relação a resistência a corrosão. Tais resultados são semelhantes aos resultados encontrados por Batista ${ }^{21}$, que mostram que quanto menor a diluição e menor o teor de ferro maior a resistência à corrosão.

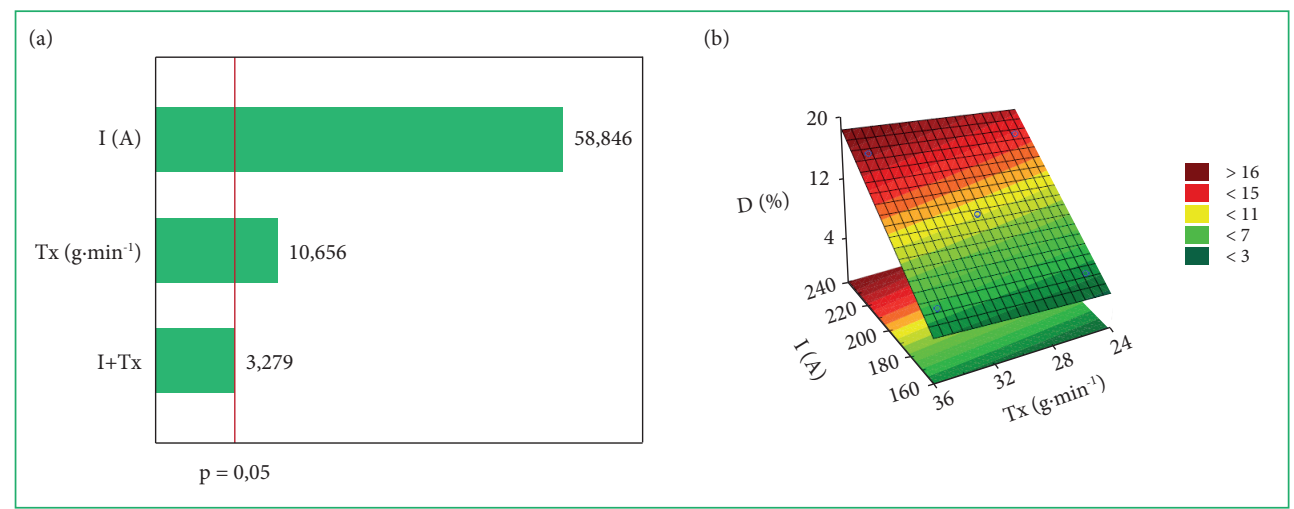

Figura 2: (a) Gráfico de Pareto para os efeitos das variáveis independentes sobre a diluição (D, \%); (b) superfície de resposta da diluição versus corrente de soldagem versus taxa de alimentação (coeficiente de determinação, $R^{2}=99,91 \%$ ).

Com relação à taxa de alimentação, os resultados da superfície de resposta (Fig. 2b) apresentam que a diluição também tende a aumentar à medida que a taxa de alimentação aumenta. Quanto maior a taxa de alimentação de pó maior corrente do arco principal requerida e maior a diluição. Porém Deuis et al. ${ }^{8}$ e Díaz ${ }^{22}$ observaram o efeito contrário, ou seja, maior taxa de alimentação de pó resulta em menor diluição, o que se deve ao fato de que maior quantidade de pó tende a retirar mais calor da poça, que é fornecido pelo processo de soldagem.

Os dados da Tabela 7 mostram que o modelo apresentado na Eq. 1 tem coeficiente de determinação $\left(R^{2}\right)$ igual a 99,91\% das variações obtidas e valor da razão de $\mathrm{F}_{\text {calculado }}$ por $\mathrm{F}_{\text {tablado }}$ igual a 127,7, indicando que o modelo é estatisticamente significativo com $95 \%$ de confiança e pode ser útil para fins preditivos.

$$
\mathrm{D}(\mathrm{I}, \mathrm{Tx})=-17,68+0,111 \times \mathrm{I}-0,191 \times \mathrm{Tx}+0,001 \times \mathrm{I} \times \mathrm{Tx} ;
$$


Tabela 7: Análise de variância para avaliação da diluição.

\begin{tabular}{|c|c|c|c|}
\hline Fator & Soma dos quadrados & Grau de liberdade & Média dos quadrados \\
\hline Regressão & 104,663 & 3 & 34,887 \\
\hline Resíduo & 0,0883 & 3 & 0,0294 \\
\hline F. ajuste & 0,085 & - & - \\
\hline Erro puro & 0,0033 & - & - \\
\hline Total & 104,751 & 6 & \\
\hline$R^{2}$ & $99,91 \%$ & & $F_{\text {calculado }} / F_{\text {tabelado }}$ \\
\hline$F_{\text {tabelado }}$ & 9,28 & & \\
\hline
\end{tabular}

\section{Influência das variáveis com relação ao teor de ferro}

De acordo com a Tabela 6, os valores de teor de ferro variaram de 3,64 a 12,88\%. Analisando os resultados obtidos na Fig. 3(a), o gráfico de Pareto demonstra que apenas a corrente de soldagem apresenta significância estatística com relação à mudança nos valores do teor de ferro, podendo-se observar que a porcentagem de teor de ferro tende a aumentar com o aumento da corrente de soldagem. É possível observar esse aumento fazendo a análise comparativa entre os pares de amostras (1 e 2) e (3 e 4), nos quais ocorre apenas a mudança na corrente de soldagem, deixando a taxa de alimentação fixa (25 e $35 \mathrm{~g} / \mathrm{min}$, respectivamente).

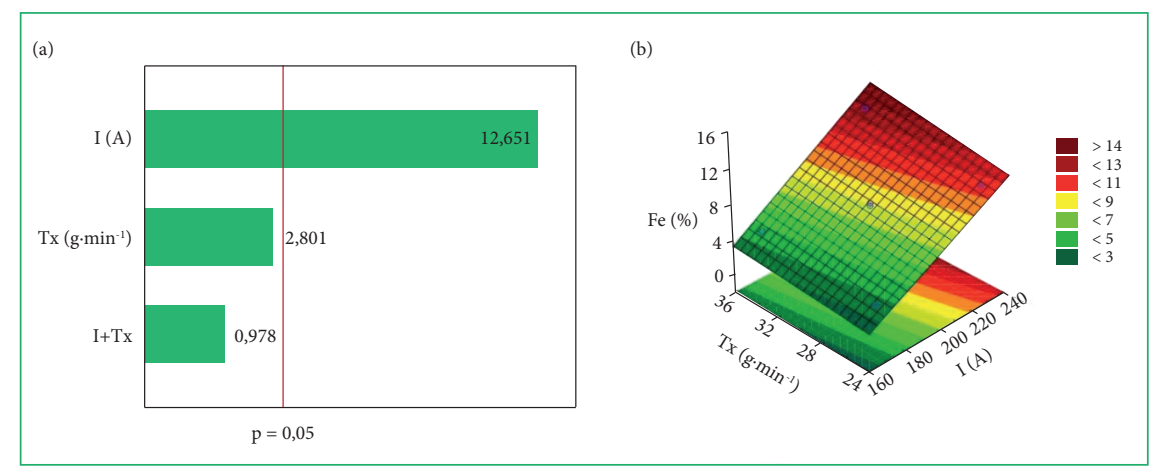

Figura 3: (a) Gráfico de Pareto para os efeitos das variáveis independentes sobre o teor de ferro (Fe, \%); (b) superfície de resposta do teor de ferro versus corrente de soldagem versus taxa de alimentação $\left(R^{2}=98,25 \%\right)$.

Esse comportamento também é comprovado na Fig. 3(b), onde a superfície de resposta obtida através da análise estatística pode ser observada. À medida que ocorre um aumento na corrente de soldagem, chega-se a teores de ferro maiores que $12 \%$. Isso é explicado pelo maior fornecimento de energia na forma de calor para poça de fusão, ou seja, maior corrente acarreta em maiores temperaturas que tendem a fundir maior quantidade de material e, portanto, maior teor de ferro estará presente na poça de fusão.

Silva et al. ${ }^{15}$ afirmam que quanto maior a diluição maior a participação de ferro e carbono no metal de solda, justificando os valores obtidos no experimento 4, no qual se obteve 16,2 e 12,88 \% para os valores de diluição e teor de ferro respectivamente. Entretanto esses valores não são desejados, pois tendem a aumentar a formação de microestruturas frágeis e suscetíveis a ataque corrosivo. Tais resultados são semelhantes aos resultados encontrados por Batista ${ }^{21}$, que mostram que quanto menor a diluição e menor o teor de ferro maior a resistência à corrosão.

Tabela 8: Análise de variância para avaliação do teor de ferro.

\begin{tabular}{|c|c|c|c|c|}
\hline Fator & Soma dos quadrados & Grau de liberdade & Média dos quadrados & $\mathbf{F}_{\text {calculado }}$ \\
\hline Regressão & 60,337 & 3 & 20,125 & 56,43 \\
\hline Resíduo & 1,072 & 3 & 0,3575 \\
\hline F. ajuste & 0,0994 & & \\
\hline Erro Puro & 0,9732 & & \\
\hline Total & 61,449 & 6 & & \\
\hline$R^{2}$ & $98,25 \%$ & & $F_{\text {calculado/ }} F_{\text {tabelado }}$ & $=6,08$ \\
\hline$F_{\text {tabelado }}$ & 9,28 & & \\
\hline
\end{tabular}


Os dados da Tabela 8 mostram que o modelo apresentado na Eq. 2 tem coeficiente de determinação ( $\left.\mathrm{R}^{2}\right)$ igual a 98,25\% das variações obtidas e valor da razão de Fcalculado por $\mathrm{F}_{\text {tabelado }}$ igual a 6,08, indicando que o modelo é estatisticamente significativo com $95 \%$ de confiança e pode ser útil para fins preditivos.

$$
\mathrm{Fe}(\mathrm{I}, \mathrm{Tx})=-10,677+0,067 \times \mathrm{I}-0,222 \times \mathrm{Tx}+0,001 \times \mathrm{I} \times \mathrm{Tx}
$$

\section{Influência das variáveis com relação à resistência à polarização}

Pode-se observar na Tabela 6 que os valores de Rp variaram de 324,58 a 486,95 K $\Omega$. A Fig. 4(a) apresenta o gráfico de Pareto, no qual observa-se que a corrente de soldagem (I) é o efeito de maior significância estatística para a variável resistência à polarização.

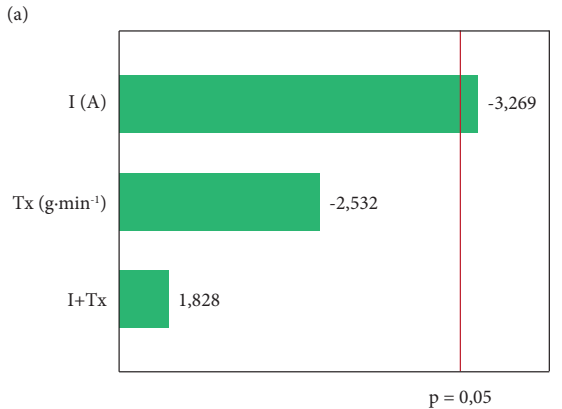

(b)

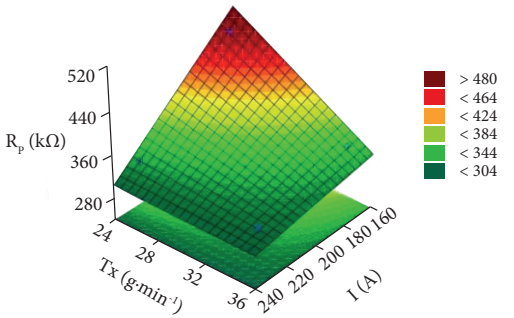

Figura 4: (a) Gráfico de Pareto para os efeitos das variáveis independentes sobre a resistência à polarização $(\mathrm{Rp}, \mathrm{K} \Omega)$; (b) superfície de resposta da resistência à polarização versus corrente de soldagem versus taxa de alimentação $\left(R^{2}=87,2 \%\right)$.

A Fig. 4(b) confirma o efeito dos parâmetros de soldagem e pode-se observar que as propriedades anticorrosivas (resistência à polarização) aumentam com a diminuição da corrente de soldagem e da taxa de alimentação, atingindo um valor máximo de aproximadamente $500 \mathrm{~K} \Omega$ (Experimento 1). Esse comportamento pode estar associado ao aporte térmico imposto durante a deposição, uma vez que ele é diretamente proporcional à corrente, ou seja, pode-se relacionar a corrente de maneira que menor corrente de soldagem resulta em menor diluição e menor teor de ferro, o que ocasiona maior resistência à polarização.

Os dados da Tabela 9 mostram que o modelo apresentado na Eq. 3 tem coeficiente de determinação ( $\mathrm{R}^{2}$ ) igual a 87,2\% das variações obtidas e valor da razão de $\mathrm{F}_{\text {calculado }}$ por $\mathrm{F}_{\text {tabelado }}$ igual a 0,73 , que indica que existe uma falta de ajuste entre as variáveis.

$$
\operatorname{Rp}(\mathrm{I}, \mathrm{Tx})=1905,238-6,640 \times \mathrm{I}-41,194 \times \mathrm{Tx}+0,17 \times \mathrm{I} \times \mathrm{Tx} ;
$$

Tabela 9: Análise de variância para avaliação da resistência à polarização.

\begin{tabular}{|c|c|c|c|c|}
\hline Fator & Soma dos quadrados & Grau de liberdade & Média dos quadrados & $\mathbf{F}_{\text {calculado }}$ \\
\hline Regressão & $16.011,74$ & 3 & $5.337,24$ & 6,815 \\
\hline Resíduo & $2.349,44$ & 3 & 783,14 \\
\hline F. Ajuste & $2.316,3$ & & \\
\hline Erro puro & 33,14 & & \\
\hline Total & 18361,18 & 6 & & \\
\hline R2 & $87,2 \%$ & & $F_{\text {calculado }} / F_{\text {tabelado }}$ & $=0,73$ \\
\hline$F_{\text {tabelado }}$ & 9,28 & & \\
\hline
\end{tabular}

\section{Influência das variáveis com relação ao potencial de corrosão}

Os valores negativos nas colunas da corrente de soldagem e da taxa de alimentação no gráfico de Pareto da Fig. 5 indicam que o aumento do potencial de corrosão é favorecido à medida que ocorre a diminuição dessas variáveis, confirmando os resultados obtidos na Tabela 6, cujos valores do potencial de corrosão variaram de $-0,562$ a - $-0,641 \mathrm{~V}$. 


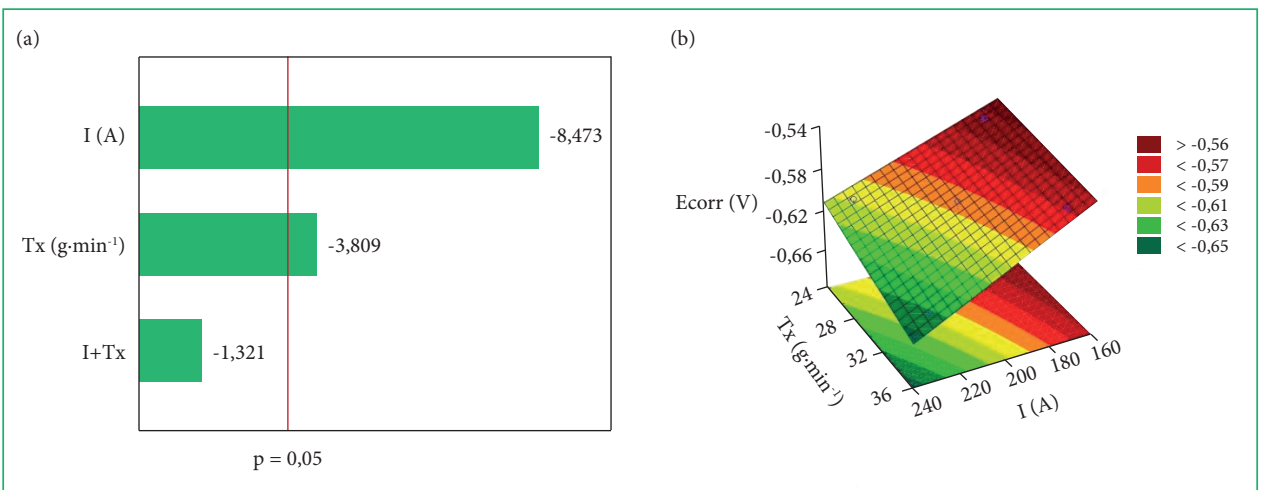

Figura 5: (a) Gráfico de Pareto para os efeitos das variáveis independentes sobre o potencial de corrosão ( $\mathrm{E}_{\text {corr, }}$ V); (b) superfície de resposta do potencial de corrosão versus corrente de soldagem versus taxa de alimentação $\left(R^{2} \stackrel{\text { corr }}{=} 96,7 \%\right)$.

A Fig. 5(b) confirma o efeito dos parâmetros de soldagem obtidos na Fig. 5(a) em função do potencial de corrosão dos revestimentos. Para valores mais próximos de zero, as propriedades anticorrosivas dos revestimentos tendem a aumentar, ou seja, o experimento 1 foi o que obteve melhores propriedades por apresentar potencial de corrosão -0,562 V.

Observa-se que a corrente de soldagem e a taxa de alimentação mostraram influência estatística significativa. Os dados da Tabela 10 mostram que o modelo apresentado na Eq. 4 tem coeficiente de determinação $\left(R^{2}\right)$ igual a 96,7\% das variações obtidas e valor da razão de $\mathrm{F}_{\text {calculado }}$ por $\mathrm{F}_{\text {tabelado }}$ igual a 3,16, indicando que o modelo é estatisticamente significativo com $95 \%$ de confiança.

$$
\text { Ecorr }(\mathrm{I}, \mathrm{Tx})=-0,512-0,00005 \times \mathrm{I}+0,003 \times \mathrm{Tx}-0,00002 \times \mathrm{I} \times \mathrm{Tx} \text {; }
$$

Tabela 10: Análise de variância para avaliação do potencial de corrosão.

\begin{tabular}{|c|c|c|c|c|}
\hline Fator & Soma dos quadrados & Grau de liberdade & Média dos quadrados & $\mathbf{F}_{\text {calculado }}$ \\
\hline Regressão & 0,00364 & 3 & 0,00121 & 29,37 \\
\hline Resíduo & 0,00012 & 3 & $4,13 \mathrm{E}-05$ \\
\hline F. Ajuste & 0,000007 & & \\
\hline Erro Puro & 0,000117 & & \\
\hline Total & 0,003767 & 6 & & \\
\hline$R^{2}$ & $96,7 \%$ & & $F_{\text {calculado }} / F_{\text {tabelado }}$ & $=3,16$ \\
\hline$F_{\text {tabelado }}$ & 9,28 & & \\
\hline
\end{tabular}

\section{Influência das variáveis em relação à corrente de corrosão}

Os valores de corrente de corrosão variam de 5,70 até 21,17 A, como pode ser observado na Tabela 6 . Realizando a análise da Fig. 6(a), pode-se observar o gráfico de Pareto em que todas as variáveis apresentaram significância estatística na variação da corrente de corrosão, ou seja, com o aumento da corrente de soldagem e taxa de alimentação, a corrente de corrosão tende a se elevar, como pode ser observado na Fig. 6(b).

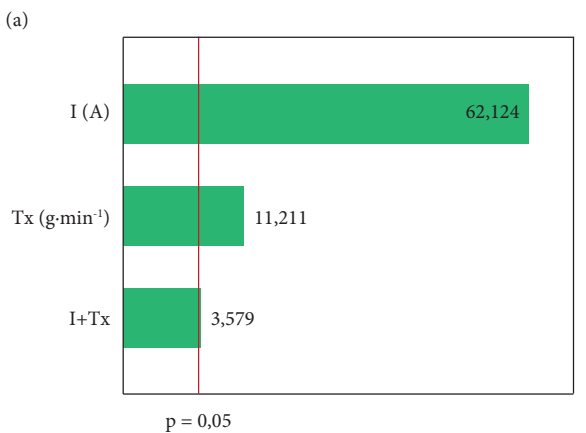

(b)

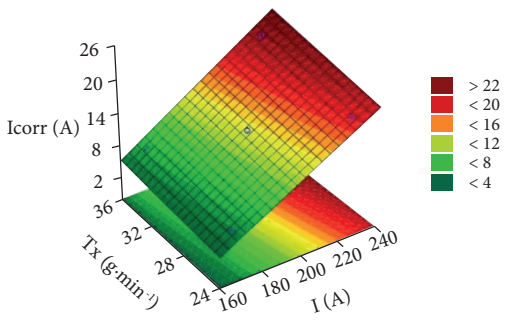

Figura 6: (a) Gráfico de Pareto para os efeitos das variáveis independentes sobre o potencial de corrosão ( $E_{\text {corr, }}$ V); (b) superfície de resposta do corrente de corrosão versus corrente de soldagem versus taxa de alimentação $\left(R^{2}=99,92 \%\right)$. 
Os dados da Tabela 11 mostram que o modelo apresentado na Eq. 5 tem coeficiente de determinação ( $\left.R^{2}\right)$ igual a 99,92\% das variações obtidas e valor da razão de $\mathrm{F}_{\text {calculado }}$ por $\mathrm{F}_{\text {tabelado }}$ igual a 143,6, indicando que o modelo é estatisticamente significativo com 95\% de confiança e pode ser útil para fins preditivos.

$$
I_{\text {corr }}(\mathrm{I}, \mathrm{Tx})=-22,504+0,142 \times \mathrm{I}-0,266 \times \mathrm{Tx}+0,002 \times \mathrm{I} \times \mathrm{Tx}
$$

Tabela 11: Análise de variância para avaliação da corrente de corrosão.

\begin{tabular}{|c|c|c|c|c|}
\hline Fator & Soma dos quadrados & Grau de liberdade & Média dos quadrados & $F_{\text {calculado }}$ \\
\hline Regressão & 177,90 & 3 & 59,301 & $1.332,61$ \\
\hline Resíduo & 0,1335 & 3 & 0,0445 \\
\hline F. Ajuste & 0,1273 & & \\
\hline Erro Puro & 0,0062 & & \\
\hline Total & 178,037 & 6 & & \\
\hline$R^{2}$ & $99,92 \%$ & & $F_{\text {calculado }} / F_{\text {tabelado }}$ & $=143,6$ \\
\hline$F_{\text {tabelado }}$ & 9,28 & & \\
\hline
\end{tabular}

A avaliação da resistência à corrosão dos revestimentos foi conduzida pela técnica de polarização potenciodinâmica linear (PPL) e, para confirmação, utilizou-se a técnica de espectroscopia de impedância eletroquímica (EIE). É possível observar na Fig. 7 as curvas de polarização potenciodinâmica dos revestimentos dos experimentos 1 e 4, os quais apresentaram a menor e a maior diluição, 4,33 e 16,2\%, respectivamente.

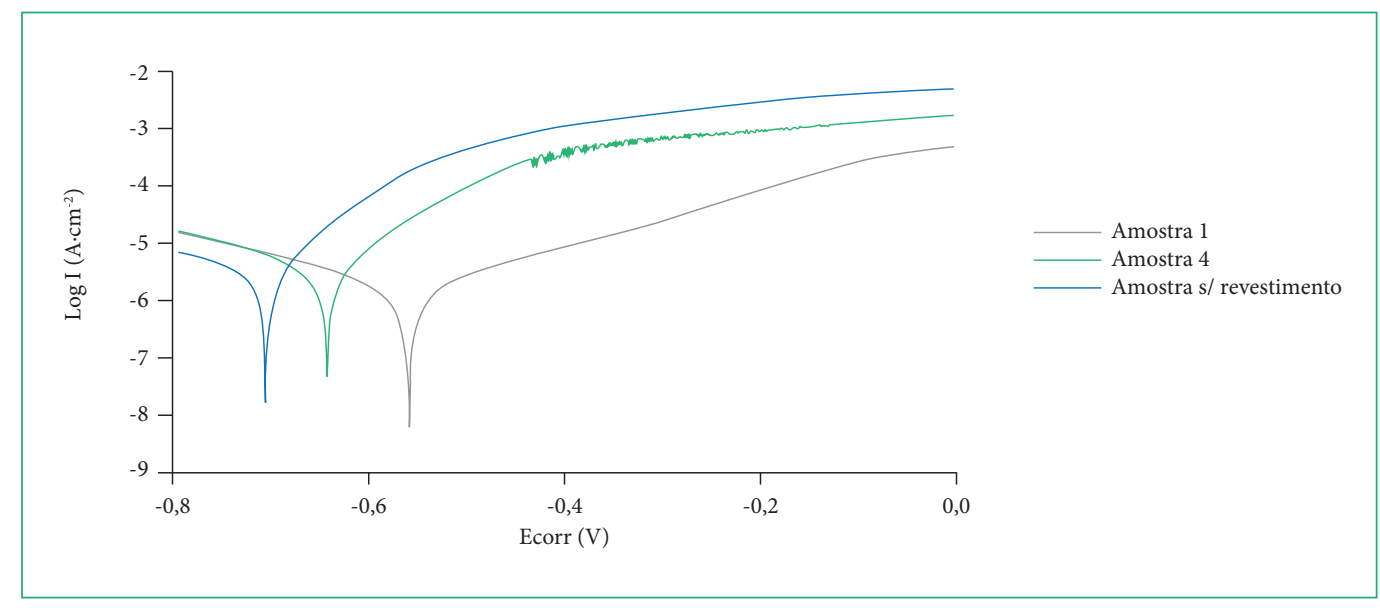

Figura 7: Curva de polarização dos experimentos com maior e menor diluição e amostra do aço sem revestimento.

O experimento 1 (com menor diluição) apresentou potencial de corrosão maior que o da amostra do experimento 4 (com maior diluição), o que indica melhores propriedades anticorrosivas, ligadas ao fato de que o primeiro experimento apresentou os seguintes resultados: teor de ferro (3,64\%) e corrente de corrosão (5,7 A) menores, o que favorece as propriedades anticorrosivas, além da resistência à polarização maior $(486,95 \mathrm{~K} \Omega$ ).

Para confirmar os resultados obtidos nas curvas de PPL sobre a resistência à corrosão foram realizadas medidas de espectroscopia de impedância eletroquímica. A Fig. 8 apresenta o diagrama de impedância dos revestimentos à base de Inconel 625 dos mesmos experimentos avaliados por PPL.

Nesse diagrama, traça-se um semicírculo que melhor se ajuste à curva a partir do eixo das abcissas e, quanto maior for o raio desse semicírculo, maior será a dificuldade pelo qual um sinal elétrico (potencial ou corrente) enviado à amostra encontrará ao percorrê-la, ou seja, maior será a resistência à corrosão do material exposto ao meio corrosivo. 


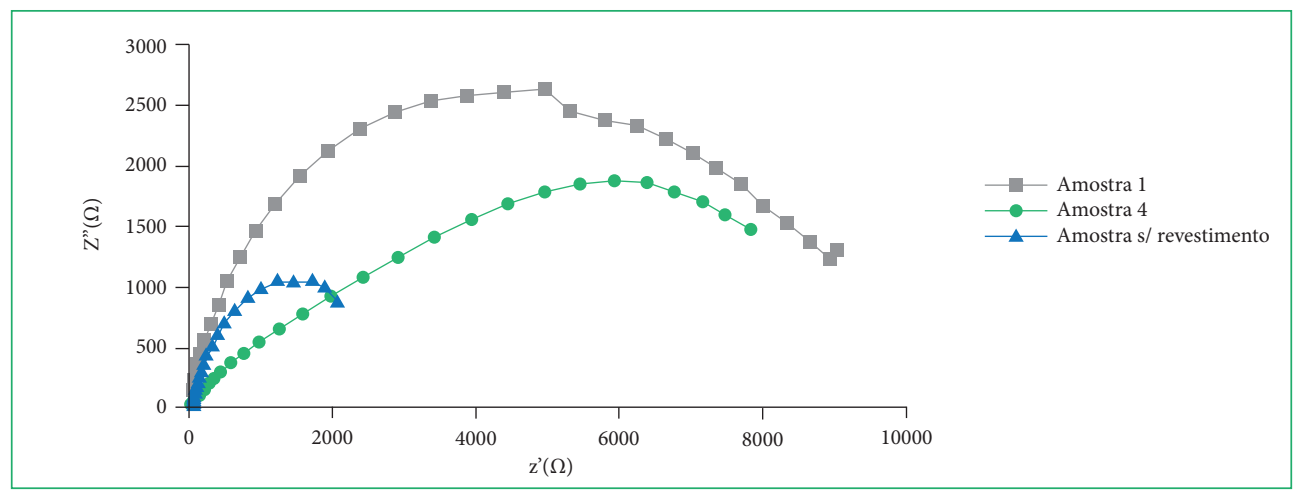

Figura 8: Curva de impedância dos experimentos com maior e menor diluição e amostra do aço sem revestimento.

\section{CONCLUSÃO}

Com base nos resultados experimentais obtidos neste trabalho, pode-se concluir que o processo de soldagem PTA-P foi utilizado com sucesso para soldagem de revestimento e que a utilização do planejamento fatorial foi satisfatória para obtenção dos melhores resultados, tanto de diluição e de composição química quanto de corrosão nos experimentos.

O experimento 1 foi o que apresentou melhor resistência à corrosão por apresentar menores valores de diluição e teor de ferro, cujos parâmetros de corrente de soldagem e taxa de alimentação foram de 170 A e $25 \mathrm{~g} / \mathrm{min}$, respectivamente. O parâmetro que apresentou maior influência estatística para o aumento da resistência à corrosão foi a corrente de soldagem, pois possui efeito direto na diluição e na composição química do revestimento.

\section{AGRADECIMENTOS}

Os autores agradecem ao Laboratório de Soldagem e Laboratório de Corrosão da Universidade Federal de Campina Grande.

\section{REFERÊNCIAS}

1. Luchtenberg P, Campos PT, Soares P, Laurindo CAH, Maranho O, Torres RD. Effect of welding energy on the corrosion and tribological properties of duplex stainless steel weld overlay deposited by GMAW/CMT process. Surf Coat Technol. 2019;375:688-93. https://doi. org/10.1016/j.surfcoat.2019.07.072

2. $[\mathrm{API}]$ American Petroleum Institute. Specification for line pipe: ANSI/API specification 5L. Chicago: American Petroleum Institute; 2007. [citado em 10 Set 2019]. Disponível em: https://www.hu-steel.com/attached/file/20160920/20160920112156975697.pdf

3. Giorgetti V, Santos EA, Marcomini JB, Sordi VL. Stress corrosion cracking and fatigue crack growth of an API $5 \mathrm{~L}$ X70 welded joint in an ethanol environment. Int J Press Vessel Pip. 2019;169:223-9. https://doi.org/10.1016/j.ijpvp.2019.01.0064

4. Callister Junior WD, Rethwisch DG. Ciência e engenharia de materiais - uma introdução. Rio de Janeiro: LTC; 2016.

5. Silva RHG, Dutra JC. Processo PTA-P-uma revisão da literatura como base para inovações. Parte 2 de 2: comportamento térmico e cinemático do pó, parâmetros e consumíveis do processo. Soldag Insp. 2012;17(2):173-83. https://doi.org/10.1590/S0104-92242012000200011

6. Reis RP, Scotti A. Fundamentos e pratica da soldagem a plasma. São Paulo: Artliber; 2007.

7. Singh $\mathrm{H}$, Jain PK. Past, present and future - the characterization of surface topography of plasma transferred arc coatings. In: 2nd Indian National Conference on Applied Mechanics. Indian: INCAM; 2015.

8. Deuis RL, Yellup JM, Subramanian C. Metal-matrix composite coatings by PTA surfacing. Compos Sci Technol. 1998;58(2):299-309. https:// doi.org/10.1016/S0266-3538(97)00131-0

9. Díaz VV, Dutra JC, D'Oliveira ASCM. Deposição por plasma com arco transferido. Soldag Insp. 2010;15(1):41-51. https://doi.org/10.1590/ S0104-92242010000100006

10. Walsh DA, Li LE, Bakare MS, Voisey KT. Visualisation of the local electrochemical activity of thermal sprayed anti-corrosion coatings using scanning electrochemical microscopy. Electrochim Acta. 2009;54(20):4647-54. https://doi.org/10.1016/j.electacta.2009.03.057

11. Sexton L, Lavin S, Byrne G, Kennedy A. Laser cladding of aerospace materials. J Mater Process Technol. 2002;122(1):63-8. https://doi. org/10.1016/S0924-0136(01)01121-9

12. Lloyd AC, Noël JJ, Mclntyre S, Shoesmith DW. Cr, Mo and W alloying additions in Ni and their effect on passivity. Electrochim Acta. 2004;49(17-18):3015-27. https://doi.org/10.1016/j.electacta.2004.01.061 
13. Liu Z, Cabrero J, Niang S, Al-Taha ZY. Improving corrosion and wear performance of HVOF-sprayed Inconel 625 and WC-Inconel 625 coatings by high power diode laser treatments. Surf Coat Technol. 2007;201(16-17):7149-58. https://doi.org/10.1016/j.surfcoat.2007.01.032

14. Suutala J, Tuominen J, Vuoristo P. Laser-assisted spraying and laser treatment of thermally sprayed coatings. Surf Coat Technol. 2006;201(5):1981-7. https://doi.org/10.1016/j.surfcoat.2006.04.042

15. Silva MM, Oliveira WC, Maciel TM, Santos MA, Motta MF. Caracterização de solda de revestimento de AWS 317L depositados por GMAW duplo arame em aços ASTM A 516 gr 60 para uso na indústria do petróleo. Soldag Insp. 2010;15(3):225-33. https://doi.org/10.1590/S010492242010000300009

16. Haeri M, Goldberg S, Gilbert JL. The voltage-dependent electrochemical impedance spectroscopy of CoCrMo medical alloy using timedomain techniques: Generalized Cauchy-Lorentz, and KWW-Randles functions describing non-ideal interfacial behaviour. Corros Sci. 2011;53(2):582-8. https://doi.org/10.1016/j.corsci.2010.09.067

17. Santos AX. Avaliação da resistência à corrosão de revestimento à base de Inconel 625 utilizando os processos de soldagem MIG e plasma pó [Dissertação]. [Campina Grande]: Universidade Federal de Campina Grande; 2014.

18. Peralta-Zamora P, Morais JL, Nagata N. Por que otimização multivariada? Eng Sanit Ambient. 2005;10(2):106-10. https://doi.org/10.1590/ S1413-41522005000200003

19. D'Oliveira ASCM, Vilar R, Feder CG. High temperature behaviour of plasma transferred arc and laser Co-based alloy coatings. Appl Surf Sci. 2002;201(1-4):154-60. https://doi.org/10.1016/S0169-4332(02)00621-9

20. Bharath RR, Ramanathan R, Sundararajan B, Srinivasan PB. Optimization of process parameters for deposition of Stellite on X45CrSi93 steel by plasma transferred arc technique. Mater Des. 2008;29(9):1725-31. https://doi.org/10.1016/j.matdes.2008.03.020

21. Batista VR. Influência dos parâmetros de soldagem sobre as características de revestimentos de liga de níquel aplicados em aços API 5L Gr B pelo processo arco submerso [Dissertação]. [Campina Grande]: Universidade Federal de Campina Grande; 2011.

22. Díaz VMV. Inovação do equipamento e avaliação do processo plasma de arco transferido alimentado com pó (PTAP) para soldagem fora de posição [Tese]. [Florianópolis]: Universidade Federal de Santa Catarina; 2005 